\title{
IFRS E PROBABILIDADE DE REPUBLICAÇÃO: UM ESTUDO DAS EMPRESAS BRASILEIRAS LISTADAS NA BOVESPA
}

\author{
IFRS AND PROBABILITY OF REPUBLISHING: A STUDY OF BRAZILIAN \\ COMPANIES LISTED ON BOVESPA
}

\author{
COSMEILSON RODRIGUES SOARES \\ Fucape Business School. Endereço: Av. Fernando Ferrari, 1358, Boa \\ Vista | 29075-505 / Vitória/ES / Brasil. \\ (1) http://orcid.org/0000-0002-3397-3015 \\ E-mail: cosmeilson@hotmail.com
}

\section{FABIO YOSHIO SUGURI MOTOKI}

Fucape Business School. Endereço: Av. Fernando Ferrari, 1358, Boa Vista / 29075-505 / Vitória/ES / Brasil.

(1) http://orcid.org/0000-0001-7464-3330

E-mail: fmotoki@fucape.br

\section{DANILO SOARES MONTE-MOR}

Fucape Business School. Endereço: Av. Fernando Ferrari, 1358, Boa Vista | 29075-505 / Vitória/ES / Brasil.

(1) http://orcid.org/0000-0002-5677-5804

E-mail:danilo@fucape.br

\section{RESUMO}

Esta pesquisa estudou o período de transição e pós-transição da adoção das Normas Internacionais para Contabilidade (International Financial Reporting Standards - IFRS) no Brasil, com o objetivo de analisar seu efeito em republicação de relatórios financeiros e na emissão de parecer de auditoria com ressalva ou reprovação. Esta pesquisa analisou 613 empresas listadas na BM\&FBovespa no período de 2001 a 2016. A análise foi elaborada por meio de um modelo Logit, testando-se as hipóteses de que a adoção das IFRS no Brasil reduzisse tanto a probabilidade de republicação quanto de emissão de parecer com ressalva ou reprovação. Os resultados obtidos indicaram que houve uma redução na probabilidade de pareceres com ressalva ou reprovação tanto no período de transição das IFRS quanto no pós-IFRS, com um efeito mais pronunciado neste último. Os resultados indicam que as IFRS podem ter tido um impacto positivo na qualidade das demonstrações, embora não tão forte quanto o desejado.

Palavras-chave: Republicação. Ressalva. IFRS.

\section{ABSTRACT}

This study analyzed the transition and post-transition period of the adoption of the International Financial Reporting Standards (IFRS) in Brazil, and its main objective was to elucidate its implications both in republishing financial reports and in issuing an accounting audit opinion, with qualification or disapproval. This research analyzed 613 companies listed on the BM\&FBovespa from 2001 to 2016. The analysis was elaborated through a Logit model, testing the hypotheses that the adoption of IFRS in Brazil would reduce both the probability of republishing and of issuing an opinion with qualification or disapproval. The results have indicated that both in the transition 
and in the post-IFRS periods there was a reduction in the probability of opinions with qualification or disapproval, with a greater effect on the latter. These results indicate that the IFRS may have impacted reporting quality positively, but not as strongly as desired.

Keywords: Republishing. Qualification. IFRS.

\section{INTRODUÇÃO}

O objetivo deste estudo é analisar o efeito das Normas Internacionais para Contabilidade (International Financial Reporting Standards - IFRS) em republicação de relatórios financeiros e na emissão de parecer de auditoria com ressalva ou reprovação. Existe alguma evidência apontando que a adoção das IFRS tem resultado na melhoria da informação contábil gerada, refletindo na apresentação mais fidedigna sobre a posição econômica e financeira de uma entidade (Ball, Robin, \& Wu, 2003; Barth, Landsman, \& Lang, 2008; Daske, Hail, Leuz, \& Verdi, 2008; Gebhardt \& Novotny-Farkas, 2011; Horton, Serafeim, \& Serafeim, 2013; Cameran, Campa, \& Pettinicchio, 2014; Lara, Torres, \& Vieira, 2008; Paananen \& Lin, 2009; Rathke, Santana, Lourenço, \& Dalmácio, 2015). Porém, como Daske et al. (2008) e Christensen, Hail e Leuz (2013) argumentam, tal resultado pode ser contingente no nível de enforcement do país. Daske, Hail, Leuz e Verdi (2013) aprofundam a investigação e encontram que empresas "sérias" na adoção de IFRS usufruem de benefícios econômicos dessa medida, enquanto as empresas "de aparência" não.

Mais recentemente, Leuz \& Wysocki (2016) expõem que a evidência dos efeitos de regulação dos relatórios contábeis é fraca, propondo mais pesquisas sobre o tema. Este trabalho se encaixa dentro desse chamado, estudando a adoção de IFRS num país de baixo enforcement como o Brasil (Durnev \& Kim, 2005). Ademais, algumas ações de melhoria não necessariamente implicam em mudanças positivas na qualidade da informação contábil, pois não levam em consideração alguns fatores importantes, tais como culturais, educacionais e jurídicos, que podem resultar em interpretações dúbias e práticas heterogêneas (Ball, 2006). Por fim, Jiang, Habib e Zhou (2015) argumentam que a evidência empírica de republicação em mercados emergentes é mínima, apesar de serem os mercados mais vulneráveis à manipulação dos relatórios contábeis. Esta investigação espera contribuir com evidência empírica sobre o tema, verificando se a adoção das IFRS no Brasil reduziu a probabilidade de republicação e de emissão de parecer de auditoria com ressalva ou reprovação, proxies para qualidade dos relatórios.

Uma das preocupações dos reguladores é manter os custos de uma nova regulação suficientemente baixos para que não anulem os potenciais benefícios da regulação. Há evidência sugerindo que a adoção das IFRS modifica a forma e o conteúdo dos relatórios contábeis, assim como o próprio processo regulatório contábil, gerando aumento de custos com a conformidade, sobretudo no que se refere à alteração dessas publicações (De George, Ferguson, \& Spear, 2013).

A partir dessas mudanças também surge a elevação dos custos de serviços de auditoria, trazendo a discussão da relação do custo-benefício do processo de convergência com as normas internacionais de contabilidade. (Hail, Leuz, \& Wysocki, 2010). Buscando medir ganhos trazidos pela adoção de IFRS, Oliveira, Dias e Gomes (2014) pesquisaram 19 empresas brasileiras com ações na bolsa de valores de Nova lorque. Os resultados indicam que houve uma diminuição de $24 \%$ nos custos de auditoria após as IFRS, fornecendo uma indicação positiva para a adoção do padrão (Oliveira et al., 2014). Vale ressaltar que o ambiente institucional brasileiro não possui uma rigorosa supervisão ou um acompanhamento do trabalho do auditor como nos Estados Unidos, o que torna importante a análise de parecer com ressalva ou reprovação (Durnev \& Kim, 2005).

Neste estudo são analisadas 613 empresas listadas na BM\&FBovespa, no período de 2001 a 2016, por meio das demonstrações contábeis e dos pareceres de auditoria, extraídos do software Economatica e do site da Comissão de Valores Mobiliários (CVM) no ano de 2017. A análise foi conduzida por meio de um modelo Logit, testando-se as hipóteses de que a adoção das IFRS no Brasil reduza tanto a probabilidade de republicação quanto a probabilidade de parecer com ressalva ou reprovação. 
Os resultados obtidos indicam que, no período de transição das IFRS, houve redução na probabilidade de pareceres de auditoria com ressalva ou reprovação, ainda que se considere 0 fato de ser um momento de adaptação ao novo paradigma contábil. Essa tendência se acentuou quando foi analisado o período pós-IFRS, pois observou-se um aumento ainda mais significativo da probabilidade de redução da ocorrência de pareceres com modificação ou reprovados. Esses resultados explicam a solidez dos relatórios contábeis em decorrência da adoção dos padrões das IFRS. Entretanto essa melhoria do ambiente informacional parece ser parcial, pois não se encontrou relação entre a adoção das IFRS e a probabilidade de republicação, abrindo um caminho para investigações que levem a um padrão superior.

Nesse prisma, espera-se que esta pesquisa contribua tanto com o meio acadêmico quanto com o mercado de capitais, com os conselhos de administração e com os acionistas e órgãos de regulação e controle sobre: (a) implicações referentes ao processo de convergência das normas internacionais de contabilidade no Brasil; (b) a adoção das IFRS e seus efeitos na probabilidade de aumento de republicações das demonstrações financeiras; e (c) seu respectivo efeito nas emissões de parecer de auditoria com ressalva.

\section{REVISÃO DE LITERATURA}

\subsection{IFRS e a qualidade da informação contábil}

A necessidade de implantação das IFRS no Brasil deriva principalmente de questões relacionadas à padronização das normas internacionais de contabilidade, bem como do incentivo ao fomento de investimentos estrangeiros no país. Uma recente pesquisa revela que mais de 25 mil entre as cerca de 48 mil companhias listadas nas 85 principais bolsas de valores do planeta adotam a padronização contábil das IFRS. Além disso, das companhias que não empregam as IFRS, mais de $80 \%$ são listadas nas bolsas da China, da Índia, do Japão e dos Estados Unidos (Girotto, 2017).

A introdução das IFRS tem influenciado as práticas contábeis, trazendo consigo um impacto significativo, sobretudo na forma como os relatórios financeiros são elaborados. $O$ padrão contábil brasileiro anterior às IFRS (os Princípios Contábeis Geralmente Aceitos - BR GAAP) foi formulado com interesses que não priorizavam informação ao mercado e, portanto, possuíam um menor nível informacional (Oliveira \& Lemes, 2011). Por sua vez, o padrão contábil internacional teve como base o fornecimento de informações que impactam as decisões estratégicas dos agentes, sobretudo visando atender às necessidades dos investidores (Cavalier \& Tiras, 2013).

Embora a adoção das IFRS em princípio tenha trazido consigo maiores exigências com relação à obtenção de uma maior qualidade da informação contábil (IFRS, 2018), ela não leva necessariamente à extinção de ações fraudulentas ou à redução dos impactos oriundos de falhas nos procedimentos contábeis (De George, Patel, \& Zeckhauser, 1999; Dechow, Ge, \& Schrand, 2010). O processo de adoção das IFRS no Brasil pode ter proporcionado maior flexibilidade no tratamento contábil, o que não existia sob os BR-GAAP, de caráter primariamente fiscal. Ao mesmo tempo em que isso permite escolhas que melhor reflitam a realidade econômicofinanceira da empresa, pode facilitar aos gestores a manipulação dos registros contábeis. (Cavalier \& Tiras, 2013).

Uma das razões da existência da auditoria é ser uma ferramenta capaz de minimizar os impactos ocasionados por conflitos de interesse (Jensen \& Meckling, 1976; Barry \& Brown, 1985; Merton, 1987). A ideia da auditoria é que um terceiro, de forma independente, assegure que a empresa apresente uma informação que reflita seus fundamentos econômico-financeiros de acordo com os GAAP. Pesquisas associam a qualidade da auditoria a diversos fatores: tamanho da firma de auditoria, seguro de auditoria, ser ou não Big Four, troca de firma de auditoria e experiência do auditor, tempo de duração do vínculo entre a empresa e a auditoria, entre outros (DeAngelo, 1981; Watts \& Zimmerman, 1981; Dye, 1993; Solomon, Shields \& Whittington, 1999; Geiger \& Raghunandan, 2002; Ghosh \& Moon, 2002; Myers, Myers \& Omer, 2003; Francis, 2011). 
Por outro lado, no que tange à associação da auditoria à qualidade da informação gerada, há uma dificuldade inerente ao se incluir a adoção das IFRS, dado que o padrão contábil é relativamente recente e é considerado um processo amplo e complexo. Apesar de existirem diversas medidas de avaliação da qualidade da informação contábil (Dechow et al., 2010), ainda não há consenso sobre o efeito da adoção das IFRS (Francis, Olsson, \& Schipper, 2006; Daske et al., 2008; Dechow et al., 2010; Horton et al., 2013; Leuz \& Wysocki, 2016; Marques, 2016). Este trabalho se insere nesse contexto, verificando se a adoção de novas normas tem relação com a republicação - um indicativo de auditoria de baixa qualidade - e com ressalva ou reprovação.

\subsection{IFRS e republicação}

A republicação dos relatórios contábeis diz respeito à divulgação de informações incorretas ou incompletas. Os pareceres expressos no relatório da auditoria podem aprovar a contabilização de valores não vistos na elaboração original do balanço. A magnitude desses valores pode motivar a republicação do balanço (Lei n. 6.404, 1976).

Diversos estudos (Kinney, Palmrose, \& Scholz, 2004; Stanley \& Dezoort, 2007; McGuire, Omer, \& Sharp, 2012; Schmidt, 2012; Francis \& Michas, 2013; Lobo \& Zhao, 2013; Jiang et al., 2015) atestam que a republicação pode ser considerada uma medida capaz de mensurar a qualidade da auditoria. Os resultados da pesquisa feita por Christensen et al. (2013) constataram que os reajustes das demonstrações contábeis são vistos como o principal indicador de má qualidade de auditoria por auditores e investidores. Pesquisas anteriores, como as de Kinney et al. (2004) e de Stanley \& Dezoort (2007), revelam distorções materiais, como ações de execução da Securities and Exchange Comission (SEC), relacionadas a relatórios financeiros fraudulentos. Porém esses casos são considerados raros e sugerem taxas muito baixas de falha de auditoria.

Existe alguma evidência de que as alterações promovidas pela adoção das IFRS no Brasil podem ter aumentado o grau de exigência dos modelos contábeis (Oliveira \& Lemes, 2011; Pelucio-Grecco, Geron, Grecco, \& Lima, 2015; Linhares, Costa, \& Beiruth, 2018). Por outro lado, a adoção de um novo modelo pode ter exigido do auditor que: (1) tenha qualificado sua mão de obra, (2) tenha trocado sua mão de obra, (3) tenha sido mais cuidadoso ou conservador por se tratar de um processo totalmente novo, ou (4) qualquer combinação dos fatores anteriores. É possível postular que essa maior qualificação ou esse maior cuidado tenham algum efeito similar ao da troca da auditoria, cuja evidência aponta para uma melhora na qualidade do relatório produzido (Silvestre, Costa \& Kronbauer, 2018). Assim, a adoção das IFRS pode resultar em uma maior incidência de republicação devido a uma auditoria mais forte. Com isso, postulamos a primeira hipótese (H1): as alterações promovidas pela adoção das IFRS no Brasil reduzem a probabilidade de republicação.

\subsection{IFRS e ressalva ou reprovação}

Existe um conflito de interesses dentro das empresas (Jensen \& Meckling, 1976; Barry \& Brown, 1985; Merton, 1987): há um incentivo para que os gestores adotem critérios de mensuração que fazem com que os demonstrativos contábeis sejam elaborados segundo seus próprios interesses e distorcendo o objetivo original de informar investidores (Becker, 1998; Chen, Chen, \& Su, 2001; Paulo, 2007).

De acordo com o American Institute of Certified Public Accountants (AICPA, 2017), o auditor deve emitir um parecer com ressalva quando:

- A evidência colhida pelo auditor indica que as demonstrações contábeis como um todo contêm distorções materiais.

- O auditor não consegue colher evidências suficientes para concluir que as demonstrações contábeis estão livres de distorções materiais

As alterações promovidas pela adoção das IFRS no Brasil podem ter aumentado o nível de complexidade dos modelos contábeis. Entretanto, a preocupação das empresas em entender e implementar corretamente o novo padrão tem o potencial de melhorar o processo de reporte significativamente, numa lógica similar ao que pode ter ocorrido com as auditorias e que levou à elaboração da H1. Isso levaria a uma diminuição do volume de emissão de pareceres com ressalva ou reprovação pelas auditorias pois, apesar de seu potencial maior escrutínio, as 
empresas tenderiam a se afastar dos requisitos citados para emissão de ressalva. Assim, temos a segunda hipótese (H2): as alterações promovidas pela adoção das IFRS no Brasil reduzem a probabilidade de ocorrência de pareceres com ressalva ou reprovação por parte das empresas de auditoria.

\section{METODOLOGIA}

Para verificar os efeitos da adoção das IFRS na probabilidade de republicação das demonstrações contábeis e de parecer de auditoria com ressalva ou reprovação das empresas brasileiras listadas na BM\&FBovespa, os dados foram coletados por meio de consulta ao software Economatica e à CVM no ano de 2017. Foram extraídos dados das empresas brasileiras listadas que divulgam as informações sobre republicações e com emissão de parecer de auditoria com ressalva ou reprovação.

A população do estudo foi o total de empresas brasileiras com ações na BM\&FBovespa. Porém foram excluídas as empresas com ausência de dados, holdings sem dados e empresas financeiras. Assim, a amostra consiste de painel não balanceado contendo 6.115 firmas-ano nas regressões de republicação e de ressalvas referentes a 613 empresas brasileiras de capital aberto listadas na BM\&FBovespa, no período de 2001 a 2016. Este estudo foi estruturado em torno do período de transição da adoção das IFRS (2008-2009) e do período pós-adoção das IFRS, com variáveis dummy para os modelos de regressão, com o intuito de mensurar se a introdução do novo padrão contábil provoca ou não mudança em relação à chance de republicação e de emissão de pareceres com ressalva.

\subsection{O modelo empírico}

Os modelos de regressão utilizados foram uma adaptação dos modelos de Gupta, Krishnan e Yu (2012). Para testar a H1, a Equação (1) estima os determinantes de republicação das demonstrações contábeis:

$\operatorname{Prob}\left(R E P_{i . t}\right)=\beta_{0}+\beta_{1} T_{-} I F R S_{i . t}+\beta_{2}$ P_IFRS $_{i . t}+\theta \operatorname{Cont}_{i . t}+\varepsilon_{i . t} \quad$ Equação (1)

A variável $R E P$, na Equação (1), é uma variável que admite dois valores: zero quando não apresenta republicação das demonstrações contábeis e um quando apresenta republicação. Como o propósito da análise consistiu em identificar se a transição das IFRS afeta a variável republicação, a variável T_IFRS assume valor um nos anos de 2008 e 2009, indicando a transição das IFRS, e zero para os demais anos. A outra variável de interesse, que afeta a

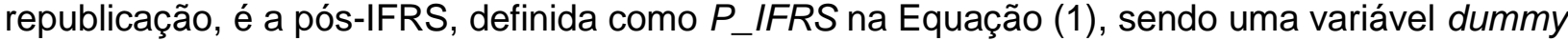
que corresponde aos valores um para os anos de 2010 a 2016 e zero para os anos de 2001 a 2007, considerados como períodos anteriores à adoção da nova norma. Cont é um vetor de variáveis de controle correspondente às variáveis definidas na Tabela $2, \operatorname{com} \theta$ indicando o vetor dos parâmetros de cada controle.

Segundo Francis, Olsson and Schipper (2006), mesmo com a adoção de normas de IFRS, não se tem conhecimento de suas reais implicações em termos de avanços da qualidade da informação contábil, tendo em vista que essas normas são relativamente recentes. Já Dechow, Ge e Schrand (2010) asseguram que inúmeras são as métricas para a verificação das características da informação financeira. Portanto outra preocupação é a de identificar os efeitos das IFRS sobre as ressalvas e as reprovações, hipótese (H2) a ser testada pela Equação (2).

$$
\operatorname{Prob}\left(\text { Res ou } \operatorname{Repr}_{i . t}\right)=\beta_{0}+\beta_{1} T_{-} I F R S_{i . t}+\beta_{2} \text { P_IFRS }_{i . t}+\theta \text { Cont }_{i . t}+\varepsilon_{i . t} \quad \text { Equação (2) }
$$

Res ou Repr é uma dummy que atua como variável resposta na Equação (2), sendo de valor um se a empresa emitiu parecer com ressalva ou reprovações no ano $t$ e zero caso contrário. Para avaliar essa variável, foram consideradas as mesmas variáveis independentes da Equação (1). 


\subsection{Definições das variáveis}

Para testar as hipóteses dos referidos modelos de regressão foi feita uma estimativa utilizando-se as variáveis dependentes e independentes descritas na Tabela 1:

Tabela 1

\begin{tabular}{|c|c|}
\hline \multicolumn{2}{|c|}{ Variáveis dependentes e independentes de interesse } \\
\hline VARIÁVEL & DEFINIÇÃO \\
\hline \multicolumn{2}{|l|}{ Variáveis independentes } \\
\hline Republicação & $\begin{array}{l}\text { Uma variável dummy definida como um se houve republicação das demonstrações } \\
\text { financeiras e zero caso contrário. }\end{array}$ \\
\hline Ressalva ou reprovação & $\begin{array}{l}\text { Uma variável dummy definida como um se a empresa recebeu um parecer com } \\
\text { ressalva ou reprovação e zero caso contrário. }\end{array}$ \\
\hline \multicolumn{2}{|r|}{ 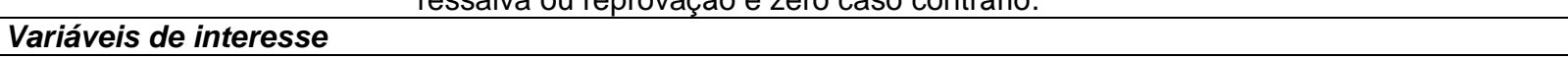 } \\
\hline Transição das IFRS & $\begin{array}{l}\text { Dummy de transição das IFRS igual a um para os anos de } 2008 \text { a } 2009 \text { e igual a zero } \\
\text { caso contrário. }\end{array}$ \\
\hline Período pós-IFRS & $\begin{array}{l}\text { Dummy de pós-IFRS igual a um para os anos de } 2010 \text { a } 2016 \text { e igual a zero caso } \\
\text { contrário. }\end{array}$ \\
\hline
\end{tabular}

Fonte: Elaborada pelo autor (2018).

Já a Tabela 2 apresenta as variáveis de controle com a intenção de se testar suas implicações em relação aos períodos de transição das IFRS e de pós-transição das IFRS.

Tabela 2

\begin{tabular}{|c|c|}
\hline VARIÁVEL DE CONTROLE & DEFINIÇÃO \\
\hline Prejuízo (LOSS) & $\begin{array}{l}\text { Uma variável dummy definida como igual a um se o lucro líquido for negativo e } \\
\text { igual a zero caso contrário. }\end{array}$ \\
\hline Alavancagem (ALAVFIN) & $\begin{array}{l}\text { Dívida de longo prazo mais a parcela corrente da dívida de longo prazo, dividida } \\
\text { pelo total de ativos. }\end{array}$ \\
\hline Tamanho (TAM) & Tamanho da empresa medido pelo logaritmo natural do valor do seu ativo total. \\
\hline Retorno sobre ativos (ROA) & Retorno sobre ativos, medido como lucro líquido dividido pelo total de ativos. \\
\hline Empresas Big Four (BIGN) & $\begin{array}{l}\text { Uma variável dummy definida como igual a um se o auditor for Big Four } \\
\text { (Deloitte, Ernst \& Young, KPMG ou PricewaterhouseCoopers) e igual a zero } \\
\text { caso contrário. }\end{array}$ \\
\hline Especialista (ESPEC) & $\begin{array}{l}\text { Uma variável de indicador igual a um se o auditor for especialista no setor, ou } \\
\text { seja, quando a auditoria for feita por uma empresa que atue em mais de um } \\
\text { segmento de firma, e igual a zero caso contrário. }\end{array}$ \\
\hline Complexidade (COMPLEX) & $\begin{array}{l}\text { Uma variável de indicador definida como igual a um se a empresa opera em } \\
\text { mais de um ramo de atividade, ou seja, em mais de um segmento de atividade } \\
\text { econômica. }\end{array}$ \\
\hline ie $t$ & Indicadores de empresa e ano, respectivamente. \\
\hline
\end{tabular}

Nota. Estas variáveis foram winsorizadas a $5 \%$.

Fonte: Elaborada pelo autor (2018).

As variáveis de controle foram definidas com base em resultados prévios da literatura (Larcker, Richardson \& Tuna, 2007; Dechow, Ge e Schrand, 2010; Cassell, Myers, Seidel, \& Zhou, 2016; Lo, Ramos e Rogo, 2017). Empresas com prejuízos (LOSS) podem ter mais dificuldades de produzir um relatório claro pois precisam justificar mais a viabilidade do negócio, o que pode ter consequências sobre a probabilidade de um evento adverso (republicação ou ressalva). A alavancagem ( $A L A V F I M)$ pode ter um efeito similar, pois significa que a empresa está mais exposta a risco financeiro. Empresas maiores (TAM) podem ter mais a explicar, estando mais sujeitas a um evento adverso. Já empresas com bom desempenho $(R O A)$ podem ter menos a explicar, gerando menores preocupações e menores chances de evento adverso. Ser auditado por uma das grandes auditorias (BIGM) pode diminuir as chances de um efeito adverso, uma vez que estas possuem mais expertise e podem ter um processo de acompanhamento da elaboração dos relatórios mais estrito, aumentando a qualidade do reporte. O mesmo efeito é esperado do fato de o auditor ser especialista (ESPEC). Já a complexidade 
da operação (COMPLEX) deve ter um efeito contrário, tornando o reporte de mais difícil elaboração e, portanto, tornando a empresa mais sujeita a eventos adversos.

\section{RESULTADOS DA PESQUISA}

\subsection{Resultados da estatística descritiva}

A Tabela 3 apresenta a estatística descritiva das variáveis aplicadas nos modelos estudados - Equações (1) e (2).

Tabela 3

\section{Estatística descritiva}

\begin{tabular}{|c|c|c|c|c|c|c|c|}
\hline Variáveis & Média & $\mathbf{D P}^{*}$ & Mínimo & Q1 & Mediana & Q3 & Máximo \\
\hline REP & 0,00 & 0,07 & 0,00 & 0,00 & 0,00 & 0,00 & 1,00 \\
\hline Res ou Repr & 0,06 & 0,23 & 0,00 & 0,00 & 0,00 & 0,00 & 1,00 \\
\hline T_IFRS & 0,13 & 0,33 & 0,00 & 0,00 & 0,00 & 0,00 & 1,00 \\
\hline $\mathrm{P}_{-}^{-}$IFRS & 0,67 & 0,47 & 0,00 & 0,00 & 1,00 & 1,00 & 1,00 \\
\hline LOOSS & 0,35 & 0,48 & 0,00 & 0,00 & 0,00 & 1,00 & 1,00 \\
\hline TAM & 12,96 & 2,88 & 5,31 & 11,58 & 13,55 & 14,98 & 17,07 \\
\hline ROA & $-0,06$ & 0,30 & $-1,14$ & $-0,03$ & 0,02 & 0,07 & 0,18 \\
\hline ALAVFIN & 1,23 & 4,99 & $-11,40$ & 0,00 & 1,40 & 2,40 & 14,50 \\
\hline ESPEC & 0,88 & 0,33 & 0,00 & 1,00 & 1,00 & 1,00 & 1,00 \\
\hline COMPLEX & 0,06 & 0,24 & 0,00 & 0,00 & 0,00 & 0,00 & 1,00 \\
\hline BIGN & 0,63 & 0,48 & 0,00 & 0,00 & 1,00 & 1,00 & 1,00 \\
\hline
\end{tabular}

Nota. *DP = desvio padrão. REP = republicação; Res ou Repr = reprovação ou ressalva; T IFRS = período de transição IFRS (20082009); P IFRS = período de pós-transição das IFRS (2010-2016); LOSS = prejuízo; TAM = tamanho da empresa; ROA = retorno sobre o ativo total; ALAVFIN = grau da alavancagem financeira; ESPEC = especialista no setor; COMPLEX = nível de complexidade; $\mathrm{BIGN}$ = se é auditada por uma Big Four. A amostra contém 613 empresas/ano listadas na Economatica e 6.115 observações, capturadas entre os anos de 2001 e 2016.

Fonte: Elaborada pelo autor (2018).

Na Tabela 3, verifica-se que as variáveis REP (republicação) e Res ou Repr (ressalva) apresentam valores baixos, pois a primeira representa apenas $0,4 \%$ da amostra (referente a 35 republicações detectadas durante o período de análise), e a segunda a $6 \%$ do total de observações. Além disso, a maior parte se encontra no período pós-IFRS ( $P_{-}$IFRS $)-67 \%$, em média -, e apenas $13 \%$ durante o período de transição (T_IFRS).

Uma parcela considerável, de 35\% das observações, apresenta prejuízo (LOSS). O forte resultado negativo é reforçado pelo $R O A$ médio negativo $(-0,06)$. Possivelmente esse cenário tem a ver com o período de estudo, que perpassa pelo período de crise de subprime (Freitas e Cintra, 2008) e pela crise brasileira (Veloso \& Bonelli, 2016; Barbosa, 2017). Em relação ao seu tamanho (TAM, total do ativo em logaritmo), as empresas são de grande porte, com uma média de $\mathrm{R} \$ 425$ milhões em ativos, sendo que a menor possui em torno de $\mathrm{R} \$ 202$ milhões em ativos. Interessante notar que a média da dívida das empresas ( $A L A V F I N)$ se encontra acima de um, o indica que elas apresentam um endividamento relativamente alto. Além disso, $88 \%$ das empresas são auditadas por especialistas, e detecta-se que poucas são complexas (COMPLEX) - $6 \%$ do total. Por fim, quase dois terços das empresas são auditadas pelas Big Four (BIGN), que, por sua vez, são consideradas as empresas de auditoria mais importantes no cenário econômico global e, teoricamente, as de mais alto padrão.

\subsection{Resultados dos testes de médias}

A Tabela 4 apresenta a comparação das médias das variáveis em estudo, buscando-se avaliar se há diferenças entre os períodos de transição e pós-IFRS. 
Tabela 4

Teste de médias

\begin{tabular}{|c|c|c|c|c|c|c|}
\hline \multirow[b]{2}{*}{ Variáveis } & \multicolumn{2}{|c|}{ Transição das IFRS ( $n=1254)$} & \multicolumn{2}{|c|}{ Período pós-IFRS (n = 4389) } & \multirow[b]{2}{*}{ Diferença } & \multirow[b]{2}{*}{ P-valor } \\
\hline & Média & Desvio padrão & Média & Desvio padrão & & \\
\hline Res ou Repr & 0,0808 & 0,2725 & 0,0392 & 0,1941 & $-0,0416$ & $0,0000^{\star \star \star}$ \\
\hline REP & 0,0058 & 0,0758 & 0,0042 & 0,0646 & $-0,0016$ & 0,3785 \\
\hline TAM & 12,7694 & 2,6662 & 13,1259 & 3,0434 & 0,3566 & $0,0000^{* * *}$ \\
\hline ROA & $-0,0437$ & 0,2750 & $-0,0702$ & 0,3135 & $-0,0264$ & $0,0005^{\star * *}$ \\
\hline ALAVFIN & 1,2188 & 5,1102 & 1,2317 & 4,8838 & 0,0130 & 0,9194 \\
\hline ESPEC & 0,8503 & 0,3568 & 0,8992 & 0,3012 & 0,0488 & $0,0000^{* * *}$ \\
\hline COMPLEX & 0,0512 & 0,2205 & 0,0664 & 0,2491 & 0,0152 & $0,0123^{\star *}$ \\
\hline BIGN & 0,5651 & 0,4958 & 0,6849 & 0,4646 & 0,1198 & $0,0000^{* * *}$ \\
\hline
\end{tabular}

Nota. REP = republicação; Res ou Repr = reprovação ou ressalva; T IFRS = período de transição IFRS (2008-2009); P_IFRS = período de pós-transição das IFRS (2010-2016); LOSS = prejuízo; TAM = tamanho da empresa; ROA = retorno sobre o ativo total; ALAVFIN = grau da alavancagem financeira; ESPEC = especialista no setor; COMPLEX = nível de complexidade; $\mathrm{BIGN}$ = se é auditada por uma Big Four, Diferença = diferença entre a média no período pós-IFRS e no período de transição;

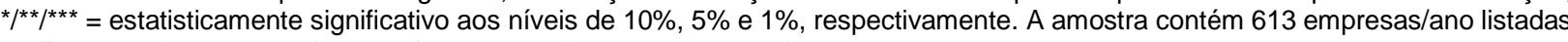
na Economatica e 6.115 observações, capturadas entre os anos de 2001 e 2016.

Fonte: Elaborada pelo autor (2018).

$\mathrm{Na}$ Tabela 4, identifica-se que as variáveis que resultaram significativas para o teste te Student foram a dependente Res ou Repr e as de controle TAM, ROA, ESPEC, COMPLEX e $B I G N$. Com isso, denota-se que as ressalvas ou reprovações (Res ou Repr) foram maiores no período de transição que no período pós IFRS, devido a uma melhora nos padrões contábeis advindos da adoção das IFRS. A diminuição de $8 \%$ para $4 \%$ da probabilidade de emissão de parecer com ressalva ou reprovação sinaliza uma melhora desse indicador, corroborando achados anteriores (Kinney et al., 2004; Stanley \& Dezoort, 2007; Mcguire et al., 2012; Schmidt, 2012; Francis \& Michas, 2013; Lobo \& Zhao, 2013).

Nota-se que o tamanho das empresas (TAM) foi maior no período pós-IFRS que no período de transição. Isso era esperado devido às novas IFRS (Hung \& Subramanyam, 2007; Pires, Decourt, Camargo, \& Siebel, 2012). Da mesma forma, identifica-se um ROA negativo, que se torna mais negativo de um período para o outro, indicando um possível efeito persistente da crise do subprime (Freitas \& Cintra, 2008) e da subsequente crise brasileira (Veloso \& Bonelli, 2016; Barbosa, 2017). A alavancagem não mostra diferença significativa. Pode-se verificar também um aumento de especialistas (ESPEC) no período pós-IFRS em relação ao de transição, o que pode ter relação com o aumento das Big Four relatado mais à frente. Já em relação à complexidade (COMPLEX), observa-se a mesma situação que foi observada na variável ESPEC: as empresas identificadas no estudo atuam em diversos segmentos no período pós-IFRS, indicando uma diversificação de suas operações. Finalmente, a variável BIGN indica que houve um aumento significativo da contratação das Big Four, o que pode estar relacionado a uma maior expertise em IFRS e a uma busca pela diminuição dos riscos de compliance.

\subsection{Resultados das regressões}

As Tabelas 5 e 6 apresentam os resultados de dois modelos de regressão, republicação (Equação 1) e ressalva (Equação 2), com o coeficiente Logit (não-linear), bem como o efeito marginal médio (uma média do efeito não-linear) e o P-valor.

Tabela 5

Regressão da variável republicação (REP)

\begin{tabular}{lcccccc}
\hline \multicolumn{1}{c}{ Variáveis } & $\begin{array}{c}\text { Coeficiente } \\
\text { Logit }\end{array}$ & Erro padrão & P-valor & $\begin{array}{c}\text { Coeficiente } \\
\text { dy/dx }\end{array}$ & Erro padrão & P-valor \\
\hline T_IFRS & 0,8722 & 0,5808 & 0,1330 & 0,0042 & 0,0028 & 0,1380 \\
P_IFRS & $-0,0437$ & 0,6871 & 0,9490 & $-0,0002$ & 0,0033 & 0,9490 \\
LOSS & 1,2500 & 0,4827 & $0,0100^{\star *}$ & 0,0060 & 0,0030 & $0,0480^{\star *}$ \\
TAM & 0,2506 & 0,1118 & $0,0250^{* *}$ & 0,0012 & 0,0007 & $0,0650^{*}$ \\
ROA & 1,2825 & 1,0167 & 0,2070 & 0,0062 & 0,0055 & 0,2560 \\
ALAVFIN & $-0,0242$ & 0,0262 & 0,3500 & $-0,0001$ & 0,0001 & 0,3770
\end{tabular}


Tabela 5 (continuação)

\begin{tabular}{lcccccc}
\hline \multicolumn{1}{c}{ Variáveis } & $\begin{array}{c}\text { Coeficiente } \\
\text { Logit }\end{array}$ & Erro padrão & P-valor & $\begin{array}{c}\text { Coeficiente } \\
\text { dy/dx }\end{array}$ & Erro padrão & P-valor \\
\hline ESPEC & 0,8204 & 0,4387 & 1,8700 & 0,0040 & 0,0021 & $0,0640^{*}$ \\
COMPLEX & 0,8111 & 0,4642 & $0,0810^{*}$ & 0,0039 & 0,0020 & $0,0500^{* *}$ \\
BIGN & $-1,6282$ & 0,4346 & $0,0000^{* * *}$ & $-0,0079$ & 0,0027 & $0,0040^{* * *}$
\end{tabular}

Nota. REP = republicação; Res ou Repr = reprovação ou ressalva; T_IFRS = período de transição IFRS (2008-2009); P_IFRS = período de pós-transição das IFRS (2010-2016); LOSS = prejuízo; TAM = tamanho da empresa; ROA = retorno sobre o ativo total; ALAVFIN = grau da alavancagem financeira; ESPEC = especialista no setor; COMPLEX = nível de complexidade; BIGN = se é auditada por uma Big Four, ${ }^{* / * * / * *}=$ estatisticamente significativo aos níveis de $10 \%, 5 \%$ e $1 \%$, respectivamente. Esta tabela descreve a Equação (1) sobre o efeito médio por Logit e o efeito marginal médio, também estimado por Logit, com erros padrão clusterizados em nível de firma. A amostra contém 613 empresas listadas na BM\&FBovespa e 6.115 observações com efeito fixo de setor, capturadas entre os anos 2001 e 2016.

Fonte: Elaborada pelo autor (2018).

A Tabela 5 descreve o coeficiente Logit (não-linear) e o efeito marginal médio de cada uma das variáveis, na probabilidade de ter ocorrido republicação. Não foram encontrados resultados estatisticamente significativos tanto no período de transição ( $T_{-}$IFRS) quanto no período pós-IFRS ( $P_{-}$IFRS). Assim, a hipótese de que talvez as auditorias tenham fortalecido o processo não se sustenta. Entretanto, esses resultados estão em linha com as ponderações de Ball (2006), ao mencionar que a adoção das IFRS não significa, necessariamente, melhoria instantânea, uma vez que aspectos culturais, políticos, entre outros, podem fazer com que as interpretações se tornem dúbias. Outro ponto a se destacar é a pouca variabilidade dos dados. Como a republicação é um evento relativamente raro, o modelo pode não estar conseguindo capturar devidamente os efeitos.

Quanto aos controles, identifica-se que as variáveis que afetam a variável republicação são as variáveis LOSS, TAM, ESPEC, COMPLEX e BIGN. Analisando os coeficientes, é possível alertar que ante uma perda (LOSS) aumenta em 0,6 p.p. a chance de republicação, indicando que empresas com perdas podem ter mais problemas em elaborar um reporte fidedigno. Como a média de republicação é baixa $(0,06 \%$ na transição e $0,04 \%$ no pós-IFRS), é um efeito econômico importante. Quanto ao tamanho da empresa (TAM), uma variação de um ponto de log no total do ativo aumenta a chance de republicar em 0,12 p.p., embora a relação estatística seja fraca. O fato de a auditoria ser especialista no setor (ESPEC) tem um efeito fracamente significante somente no efeito marginal médio, com um aumento de 0,40 p.p. na chance de republicação. Firmas mais complexas, que atuam em mais de um setor (COMPLEX), também apresentam uma chance estatisticamente significante de maior republicação, de $0,2 \%$, provavelmente devido à maior dificuldade inerente de reportar a operação. Por fim, ser auditado por uma Big Four (BIGM) está associado a uma possibilidade 0,79 p.p. menor de republicação, indicando uma maior qualidade do processo de auditoria. $R O A$ e alavancagem parecem não estar relacionados com a chance de republicação.

A Tabela 6 apresenta a regressão da variável ressalva estimada por Logit e também pelo coeficiente $(\mathrm{dy} / \mathrm{dx})$, apresentando o efeito marginal médio:

Tabela 6

Regressão da variável ressalva ou reprovação (RES ou REPR)

\begin{tabular}{lcccccc}
\hline Variáveis & $\begin{array}{c}\text { Coeficiente } \\
\text { Logit }\end{array}$ & Erro padrão & P-valor & $\begin{array}{c}\text { Coeficiente } \\
\text { dy/dx }\end{array}$ & Erro padrão & P-valor \\
\hline T_IFRS & $-0,4319$ & 0,1632 & $0,0008^{* * *}$ & $-0,0227$ & 0,0073 & $0,0002^{* * *}$ \\
P_IFRS & $-0,8640$ & 0,1949 & $0,0000^{* * *}$ & $-0,0454$ & 0,0120 & $0,0000^{* * *}$ \\
LOSS & 1,0484 & 0,1351 & $0,0000^{* * *}$ & 0,0551 & 0,0110 & $0,0000^{* * *}$ \\
TAM & 0,0805 & 0,0640 & 0,2090 & 0,0042 & 0,0034 & 0,2120 \\
ROA & 0,1084 & 0,4157 & 0,7940 & 0,0057 & 0,0222 & 0,7970 \\
ALAVFIN & $-0,0059$ & 0,0134 & 0,6580 & $-0,0003$ & 0,0007 & 0,6550 \\
\hline
\end{tabular}


Tabela 6 (continuação)

\begin{tabular}{lcccccc}
\hline Variáveis & $\begin{array}{c}\text { Coeficiente } \\
\text { Logit }\end{array}$ & Erro padrão & P-valor & $\begin{array}{c}\text { Coeficiente } \\
\text { dy/dx }\end{array}$ & Erro padrão & P-valor \\
\hline ESPEC & $-0,1848$ & 0,5016 & 0,7130 & $-0,0097$ & 0,0263 & 0,7120 \\
COMPLEX & 0,2534 & 0,5455 & 0,6420 & 0,0133 & 0,0281 & 0,6360 \\
BIGN & $-0,7692$ & 0,2277 & $0,0001^{* * *}$ & $-0,0405$ & 0,0141 & $0,0004 * * *$
\end{tabular}

Nota. REP = republicação; Res ou Repr = reprovação ou ressalva; T_IFRS = período de transição IFRS (2008-2009); P_IFRS = período de pós-transição das IFRS (2010-2016); LOSS = prejuízo; TAM = tamanho da empresa; ROA = retorno sobre o ativo total; ALAVFIN = grau da alavancagem financeira; ESPEC = especialista no setor; COMPLEX = nível de complexidade;

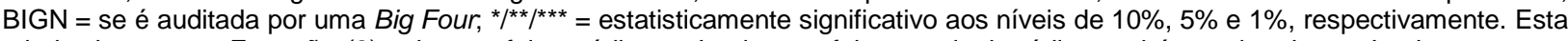
tabela descreve a Equação (2) sobre o efeito médio por Logit e o efeito marginal médio, também estimado por Logit, com erros padrão clusterizados em nível de firma. A amostra contém 613 empresas listadas na BM\&FBovespa e 6.115 observações com efeito fixo de setor, capturadas entre os anos 2001 e 2016

Fonte: Elaborada pelo autor (2018).

A Tabela 6 indica que foram encontrados resultados estatisticamente significativos nos períodos de transição ( $T$ IIFRS) e pós-IFRS ( $P_{-}$IFRS), suportando a H2. Durante a transição observa-se uma redução de 2,27 p.p. na probabilidade de emissão de pareceres de auditoria com ressalva ou reprovação (Res ou Repr), com um efeito econômico ainda mais pronunciado no período pós-IFRS, em que houve redução de 4,54 p.p. É um efeito econômico importante quando contrastado com as médias de $8 \%$ e $4 \%$ nos períodos de transição e pós-IFRS. Portanto existe alguma indicação de que os relatórios contábeis tiveram uma melhora em sua qualidade, corroborando resultados obtidos em outros cenários, tanto no Brasil quanto no exterior (Oliveira \& Lemes, 2011; Pelucio-Grecco et al., 2015; Linhares, Costa \& Beiruth, 2018)

Em termos de controles, LOSS continua com resultado altamente significativo, evidenciando que as perdas também possuem um efeito importante sobre a chance de ressalva ou reprovação (Res ou Repr), com um aumento de 5,51 p.p. Ser auditado por uma Big Four continua tendo um resultado similar sobre ressalvas e reprovações, com uma diminuição de 4 p.p. na chance de ocorrência de problemas desse tipo. Os demais controles mostram-se não significativos.

Assim, há alguma evidência de que talvez a adoção das IFRS não tenha tido um impacto tão forte nas demonstrações contábeis, diante do não resultado em republicações que demonstrou este estudo. Tal resultado está em linha com as afirmações de Ball et al. (2003) de que os incentivos econômicos dominam os padrões contábeis na determinação da qualidade das demonstrações financeiras. Entretanto há evidência de que alguma melhora pode ter ocorrido, uma vez que no período posterior às IFRS diminuiu o volume de pareceres ressalvados ou reprovados.

\section{CONCLUSÃO}

Este estudo objetivou analisar o efeito das Normas Internacionais para Contabilidade (International Financial Reporting Standards - IFRS) em republicação de relatórios financeiros e na emissão de parecer de auditoria com ressalva ou reprovação. Para responder à questão da pesquisa, foi utilizada uma amostra coletada por meio de base de dados da Economatica e da CVM. Foram estudadas 613 empresas de capital aberto, listadas na BM\&FBovespa, no período de 2001 a 2016.

Os resultados deste estudo indicam um efeito positivo da adoção das IFRS na qualidade das demonstrações contábeis brasileiras ao evidenciar diminuição da ocorrência de ressalvas e reprovações por parte das empresas de auditoria. Entretanto não se observou nenhum efeito relativo à chance de republicação, ou seja, embora as empresas de auditoria estejam ressalvando ou reprovando menos os balanços, não se verifica redução na probabilidade de republicação destes. Nessa perspectiva, os resultados desta pesquisa podem ser úteis para reguladores como a CVM, o Comitê de Pronunciamentos Contábeis (CPC) ou o próprio International Accounting Standards Board (IASB) - que tem a função de trazer transparência, accountability e eficiência para os mercados financeiros -, na medida em que sinaliza uma melhora apenas parcial na qualidade das demonstrações contábeis.

Este estudo, por estar limitado às empresas listadas, não analisa o universo de empresas fechadas e auditadas. Por envolver empresas fechadas e com menores requisitos de disclosure, é possível que os efeitos das IFRS pudessem ser ampliados caso tais empresas fossem 
analisadas. Ou seja, sugere-se que pesquisas futuras verifiquem os efeitos da adoção das normas internacionais para além do universo de empresas listadas. Por fim, é válido enfatizar que existem oportunidades para novos estudos, pesquisando, por exemplo, outras formas de verificação da adoção das IFRS, a saber: i) quais são as organizações com uma relação custo/benefício mais atraente; ii) qual pode ser o efeito da adoção das IFRS no uso da informação contábil por outros usuários; iii) verificar minuciosamente se as empresas de fato adotam as IFRS, ou seja, analisar o grau de cumprimento das novas regras estabelecidas por esse paradigma contábil; entre outros.

\section{REFERÊNCIAS}

AICPA (2017). Modifications to the Opinion in the Independent Auditor's Report. Recuperado de https://www.aicpa.org/research/standards/auditattest/downloadabledocuments/au-c00705.pdf

Ball, R. (2006). International Financial Reporting Standards (IFRS): pros and cons for investors. Accounting and Business Research, 36(1), 5-27. doi: 10.1080/00014788.2006.99730040

Ball, R., Robin, A., \& Wu, J. S. (2003). Incentives versus standards: properties of accounting income in four East Asian countries. Journal of Accounting and Economics, 36(1), 235-270. doi: https://doi.org/10.1016/j.jacceco.2003.10.003

Barbosa, F. de H., Filho (2017). A crise econômica de 2014/2017. Estudos Avançados, 31(89), 51-60.

Barry, C. B. \& Brown, S. J. (1985). Differential information and security market equilibrium. Journal of Financial and Quantitative Analysis, 20(4), 407-422.

Barth, M. E., Landsman, W. R., \& Lang, M. H. (2008). International Accounting Standards and Accounting Quality. Journal of Accounting Research, 46(3), 467-498. doi: 10.1111/j.1475679X.2008.00287.x

Becker, C. L., DeFond, M. L., Jiambalvo, J., \& Subramanyam, K. R. (1998). The effect of audit quality on earnings management. Contemporary accounting research, 15(1), 1-24.

Cameran, M., Campa, D., \& Pettinicchio, A. (2014). IFRS Adoption Among Private Companies: Impact on Earnings Quality. Journal of Accounting, Auditing \& Finance, 29(3), 278-305. doi: $10.1177 / 0148558 \times 14534260$

Cassell, C. A., Myers, L. A., Seidel, T. A., \& Zhou, J. (2016). The Effect of Lame Duck Auditors on Management Discretion: An Empirical Analysis. Auditing: A Journal of Practice \& Theory, 35(3), 51-73. doi: https://doi.org/10.2308/ajpt-51378

Cavalier, G. R. \& Tiras, S. L. (2013). Brazil's adoption of IFRS: fertile ground for examining earnings management. Brazilian Business Review, 10(4), 133.

Chen, C. J. P., Chen, S., \& Su, X. (2001). Profitability Regulation, Earnings Management, and Modified Audit Opinions: Evidence from China. AUDITING: A Journal of Practice \& Theory, 20(2), 9-30.

Christensen, H. B., Hail, L., Leuz, C. (2013), Mandatory IFRS reporting and changes in enforcement. Journal of Accounting and Economics, 56, 147-177. doi: 10.1016/j.jacceco.2013.10.007

Daske, H., Hail, L., Leuz, C. \& Verdi, R. (2008). Mandatory IFRS Reporting around the World: Early Evidence on the Economic Consequences. Journal of Accounting Research, 46, 
1085-1142. doi: 10.1111/j.1475-679X.2008.00306.x

Daske, H., Hail, L., Leuz, C., \& Verdi, R. (2013). Adopting a Label: Heterogeneity in the Economic Consequences Around IAS/IFRS Adoptions. Journal of Accounting Research, 51, 495-547. doi: 10.1111/1475-679X.12005

De George, E. T., Ferguson, C. B., \& Spear, N. A. (2012). How much does IFRS cost? IFRS adoption and audit fees. The Accounting Review, 88(2), 429-462.

De George, F., Patel, J., \& Zeckhauser, R. (1999). Earnings management to exceed thresholds. The Journal of Business, 72(1), 1-33.

DeAngelo, L. E. (1981). Auditor independence, 'low balling', and disclosure regulation. Journal of accounting and Economics, 3(2), 113-127.

Dechow, P., Ge, W., \& Schrand, C. (2010). Understanding earnings quality: A review of the proxies, their determinants and their consequences. Journal of accounting and economics, 50(2-3), 344-401.

Durnev, A. \& Kim, E. H. (2005). To steal or not to steal: Firm attributes, legal environment, and valuation. The Journal of Finance, 60, 1461-1493.

Dye, R. A. (1993). Auditing standards, legal liability, and auditor wealth. Journal of political Economy, 101(5), 887-914.

Francis, J. R. (2011). A framework for understanding and researching audit quality. Auditing: A journal of practice \& theory, 30(2), 125-152.

Francis, J. R., \& Michas, P. N. (2013). The contagion effect of low-quality audits. The Accounting Review, 88(2), 521-552.

Francis, J., Olsson, P., \& Schipper, K. (2006). Earnings Quality: fundations and trends in accounting. Boston: Now Publisher Inc.

Freitas, M. C. P. D., \& Cintra, M. A. M. (2008). Inflação e deflação de ativos a partir do mercado imobiliário americano. Revista de Economia Política, 28(3).

Gebhardt, G., \& Novotny-Farkas, Z. (2011). Mandatory IFRS Adoption and Accounting Quality of European Banks. Journal of Business Finance \& Accounting, 38(3-4), 289-333. doi: https://doi.org/10.1111/j.1468-5957.2011.02242.x

Geiger, M. A. \& Raghunandan, K. (2002). Auditor tenure and audit reporting failures. Auditing: A Journal of Practice \& Theory, 21(1), 67-78.

Ghosh, A. \& Moon D. (2002). Does Auditor Tenure Impair Audit Quality? Working paper, Baruch College.

Girotto, M. (2017). Pesquisas apontam os resultados da adoção das normas IFRS no Brasil. Revista Brasileira de Contabilidade, (222), 6-15. Recuperado de http://rbc.cfc.org.br/index.php/rbc/article/view/1482

Gupta, P., Krishnan, G., \& Yu, W. (2012). Do Auditors Allow Earnings Management When Audit Fees Are Low? Recuperado de http://ssrn.com/abstract=1836829. doi: $10.2139 /$ ssrn. 1836829

Hail, L., Leuz, C., \& Wysocki, P. D. (2010). Global Accounting Convergence and the Potential Adoption of IFRS by the U.S. (Part I): Conceptual Underpinnings and Economic Analysis. 
Accounting Horizons, 24(3), 355-394.

Horton, J., Serafeim, G., \& Serafeim, I. (2013). Does Mandatory IFRS Adoption Improve the Information Environment?. Contemporary Accounting Research, 30, 388-423. doi: 10.1111/j.1911-3846.2012.01159.x

Hung, M. \& Subramanyam, K. R. (2007). Financial statement effects of adopting international accounting standards: the case of Germany. Review of accounting studies, 12(4), 623-657.

IFRS (2018). Why global accounting standards? Recuperado em 25 de setembro de 2018 de https://www.ifrs.org/use-around-the-world/why-global-accounting-standards/.

Jensen, M. \& Mackling, W. H. (1976). Theory of The Firm: Managerial Behavior, Agency Cost and Ownership Structure. Journal of Financial Economics.

Jiang, H., Habib, A., \& Zhou, D. (2015) Accounting restatements and audit quality in China. Advances in Accounting, 31, 125-135. doi: 10.1016/j.adiac.2015.03.014

Kinney, W. R., Palmrose, Z. V., \& Scholz, S. (2004). Auditor Independence, Non-Audit Services, and Restatements: Was the US Government Right?. Journal of Accounting Research, 42(3), 561-588.

KPMG (2009). A fraude no Brasil. Relatório da pesquisa 2009. Recuperado em 04 março, 2016, de http://www.kpmg.com.br/publicacoes/forensic/fraudes_2009_port.pdf

Lara, J. M. G., Torres, J. A. R., \& Veira, P. J. V. (2008). Conservatism of earnings reported under International Accounting Standards: A comparative study. Spanish Journal of Finance and Accounting, 37(138), 197-210. doi: https://doi.org/10.1080/02102412.2008.10779642

Larcker, D. F., Richardson, S. A., \& Tuna, I. (2007). Corporate Governance, Accounting Outcomes, and Organizational Performance. The Accounting Review, 82(4), 963-1008. doi: https://doi.org/10.2308/accr.2007.82.4.963

Lei n. 6.404, de 15 de dezembro de 1976 (1976). Recuperado 23 de outubro de 2018, de http://www.planalto.gov.br/ccivil_03/LEIS/L6404consol.htm

Leuz, C. \& Wysocki, P. D. (2016). The Economics of Disclosure and Financial Reporting Regulation: Evidence and Suggestions for Future Research. Journal of Accounting Research, 54, 525-622. doi: 10.1111/1475-679X.12115

Linhares, F., Costa, F., \& Beiruth, A. (2018). Earnings management and investment efficiency. Review of Business Management, 20(2), 295-310. doi: 10.7819/rbgn.v20i2.3180

Lo, K., Ramos, F., \& Rogo, R. (2017). Earnings management and annual report readability. Journal of Accounting and Economics, 63(1), 1-25. doi: https://doi.org/10.1016/j.jacceco.2016.09.002

Lobo, G. J. \& Zhao, Y. (2013). Relation between audit effort and financial report misstatements: Evidence from quarterly and annual restatements. The Accounting Review, 88(4), 13851412.

Marques, V. (2016). Qualidade das Informações Financeiras e o Ambiente Regulatório: Evidências empíricas no Período de 1998-2013. Tese de Doutorado em Administração, Centro de Pós-Graduação e Pesquisas em Administração - CEPEAD, Universidade Federal de Minas Gerais. Belo Horizonte, MG, Brasil.

McGuire, S. T., Omer, T. C., \& Sharp, N. Y. (2012). The impact of religion on financial reporting 
irregularities. The Accounting Review, 87(2), 645-673.

Merton, R. C. (1987). A simple model of capital market equilibrium with incomplete information. The journal of finance, 42(3), 483-510.

Myers, J. N., Myers, L. A., \& Omer, T. C. (2003). Exploring the term of the auditor-client relationship and the quality of earnings: A case for mandatory auditor rotation? The Accounting Review, 78(3), 779-799.

Oliveira, F. S., Dias, J. M., Filho, \& Gomes, S. M. da S. (2014). O Impacto do IFRS sobre os Custos de Auditoria no Brasil 2014. Anais do Encontro da ANPAD, Rio de Janeiro, RJ, Brasil, 38.

Oliveira, V. \& Lemes, S. (2011). Nível de convergência dos princípios contábeis brasileiros e norte-americanos às normas do IASB: uma contribuição para a adoção das IFRS por empresas brasileiras. Revista Contabilidade \& Finanças, 22(56), 155-173. doi: $10.1590 / \mathrm{S} 1519-70772011000200003$

Paananen, M., \& Lin, H. (2009). The Development of Accounting Quality of IAS and IFRS over Time: The Case of Germany. Journal of International Accounting Research, 8(1), 31-55. doi: https://doi.org/10.2308/jiar.2009.8.1.31

Paulo, E. (2007). Manipulação das informações contábeis: uma análise teórica e empírica sobre os modelos operacionais de deteçã̃o de gerenciamento de resultados. Tese de Doutorado, Universidade de São Paulo, São Paulo, Brasil.

Pelucio-Grecco, M. C., Geron, C. M. S., Grecco, G. B. \& Lima, J. P. C. (2014). The effect of IFRS on earnings management in Brazilian non-financial public companies. Emerging Markets Review, 21, 42-66. doi: 10.1016/j.ememar.2014.07.001

Pires, C. de O., Decourt, R. F., Camargo, C. U. de O., \& Siebel, V. (2012). Os impactos da fase final de transição para o IFRS no Brasil. Anais do Seminários em Administração, São Paulo, SP, Brasil, 15.

Rathke, A. A. T., Santana, V. de F., Lourenço, I. M. E. C., \& Dalmácio, F. Z. (2016). International Financial Reporting Standards and Earnings Management in Latin America. Revista de Administração Contemporânea, 20(3), 368-388. doi: https://doi.org/10.1590/19827849rac2016140035

Schmidt, J. J. (2012). Perceived auditor independence and audit litigation: The role of nonaudit services fees. The Accounting Review, 87(3), 1033-1065.

Silvestre, A. O., Costa, C. M., \& Kronbauer, C. A. (2018). Audit Rotation And Earnings Quality: An Analysis Using Discretionary Accruals. Brazilian Business Review, 15(5), 410-426. doi: 10.15728/bbr.2018.15.5.1

Solomon, I., Shields, M. D., \& Whittington, O. R. (1999). What do industry-specialist auditors know? Journal of accounting research, 37(1), 191-208.

Stanley, J. D., \& DeZoort, F. T. (2007). Audit firm tenure and financial restatements: An analysis of industry specialization and fee effects. Journal of Accounting and Public Policy, 26(2), 131-159.

Veloso, F. \& Bonelli, R. (2016). A Crise de Crescimento do Brasil. São Paulo: Elsevier.

Watts, R. L. \& Zimmerman, J. L. (1981). The markets for independence and independent auditors. Unpublished manuscript. University of Rochester, NY. 